\title{
User Involvement in System Development Process
}

\author{
Zhiwei Sun \\ Department of Information Science and Technology \\ Tianjin University of Finance \& Economics \\ Tianjin, China \\ email:Sunzhiwei_0@163.com
}

\begin{abstract}
User involvement is playing a more and more important role in the system developing cycle.Meaningful user involvement in systems development and an overall user orientation is critical to the success of any development project. This paper will concern with the definition of user involvement, why it is considered desirable and how it works in the system development methodology. Furthermore, it will compare user involvement in two system development methodologies: SSM and SSADM.
\end{abstract}

Keywords-User involvemen; System development; SSM; SSADM

\section{INTRODUCTION}

In the field of information system, user involvement is a significant aspect in the system development cycle. For the past twenty years, user involvement has been accepted as an ingredient in the success of systems development efforts (Barki \& Hartwick, 1989). If users involve into the system development cycle, they can give more information details. As Dodd \& Carr (1994) stated users are often ancillaries in this development environment, whereas data services only develop the system in isolation.' Therefore one widely understanding of user involvement is really helpful for defining system requirements in real world and ensuring system methodology implementation efficient.

There is an increasing need to involve users into the software development life-cycle. In recent studies of software projects (Herlea, 1999), user involvement, management support and clear statements of requirements are thought as major reasons in leading system development to succeed. The lack of effective user input will cause incomplete requirements and specifications.

In this paper, it will concern with the definition of user involvement, why it is considered desirable and how it works in the system development methodology. Furthermore, it will compare user involvement in two system development methodologies: SSM and SSADM.

\section{DEFINITION OF USER INVOLVEMENT}

The concept of user involvement in the development process had its genesis in the period of 1970's. Initially the system researchers focused on "sociotechnical" systems that meant the joint optimization of both technical and social aspects of system design (Fitzgerald, 1997). In the information system, user involvement generally referred to "participation in the system development system development process by potential users or representatives” (Barki \& Hartwick, 1989). However, Barki \& Hartwick (1989) proposed to define user involvement as a subjective psychological state that thought a user was involved when the user believes that the system was significant and personally relevant.

Additionally it is hard to define the term of 'user'. Carmel, Whitaker and George (1993) believe that an unambiguous definition of user is impossible. Some of developers think that user refers to any non-IS or nontechnical individual in the organisation who is affected by the system. It includes managers. While others understand "user" as any operational worker who is affect by system. It does not include managers. In this paper, it assumes that user means the person who participates in developing the new system.

Research finds that "users' involvement in design and implementation is related positively to users' perception of system usefulness" (Franz \& Robey, 1986). Furthermore, "active participation by users in the design process instills a sense of ownership in the final product, which produces a number of benefits, like increased willingness to use the system, better system design, and a more positive attitude to computer systems" (Copenhaver, 1986). Thus, effective user involvement can contribute a better understanding to the system development, and provide satisfactory product as well.

\section{IMPORTANCE OF USER INVOLVEMENT}

User involvement is a significant aspect in the system development. Many methodologies concern with users in the development phases. It embodies the view of users, not systems analysts, programmers, or the data services organization (Dodd \& Carr, 1994). It has several important aspects in information system development.

\section{A. Why user involvement is considered desirable}

Firstly, in the system development, especially in the initial (feasibility) study and design phases, system developers need to find out the existing problems and identify the requirements for the new system. Because usually except system developers who have worked in the current system for a long period, most developers aren't familiar with the current system, they can't have a deep understanding of the system environment. Consequently if they want to understand the current system, a good way is to communicate with the end-users of the current system. Beyer 
\& Holtzblatt (1995) claim that users are experts in their work and a thorough understanding of the requirements is reached only by promoting effective communication with them during the requirements definition process. It is helpful to establish a channel between the users and system developers for exchanging the information. However, because of limited fund and time requirements, it is impossible to involve all users in the process. Consequently, system developers have to select users carefully aiming to get most valuable information.

Secondly, user involvement can help the system developers to get fast and easy methodologies (Allen, Ballman, Begg, et al., 1993). It means system developers can assess and certify data from the secondary sources to identify users' requirements, thus, system developers can apply more appropriate methodology. Furthermore, user involvement can lead to the simpler methods to design and validate the system software functionality.

Thirdly, user involvement can provide the "more reliable ways to organize features into menus and dialog boxes based on user data" (Allen, Ballman, Begg, et al., 1993). In the earlier system development methodologies, system developers seldom to consider the users' working habits, when the new system is applied in the environment, endusers always find it is hard to manipulate although system developers believe the system is reliable and easy to use. User involvement can reduce or even eliminate the conflict between users and system developers in the system function views. By communicating with users, system developers find data and information direct from the users; understand needs of the users and help users have an initial understanding of the new system. After further negotiation with the users, system developers and users can get consensus on the new system.

Finally, user involvement enables the system developers to know and understand the user's lexicon so the system developers can communicate using the same language (Allen, Ballman, Begg, et al., 1993). For system developers, especially those who are outside the system environment, they don't have experience of current system. However users can be considered as the experts of the current system environment, so developers can know ordinary language used in the environment from users. If system developers can use the same "language" to communicate with the users, system development efficiency can be sped up.

\section{B. The advantages of user involvement}

Dodd \& Carr (1994) consider that user is the first member of a team organized to define problems. Users may participate data gathering, data flow diagrams reviewing, and use prototyping. Adoption of user involvement can have several benefits for system development methodologies:

- User involvement helps system developers identify the current problems that might be neglected because lack of environment understanding.

- $\quad$ As stated by Dodd \& Carr (1994), user involvement can avoid the conflict between users and data services. Temporarily ignoring the conflict between the continuity of system development cycle and the limitation of the budget and time, user should be involved throughout the system development activities. In this way system developers can communicate with them at any time for meeting latest needs and improve the data service.

- "Jointly involving users and systems professionals helps create an understanding of why trade-offs are made" (Dodd \& Carr, 1994). If the systems professionals don't communicate with the users, it is very possible for users to complain that the delivered system doesn't fit with their requirements although they believe that product is appropriate. However if users are involved into the whole development process and are encouraged to communicate with developers, they can reveal the existing problem situation better. Meanwhile, during development process, users can give immediate feedback when they find any mismatch between system design and expected requirements. In these ways, the trade-offs can be more reliable and reasonable.

- User involvement improves the overall computer literacy of the company and provides better understanding of computer-based technology and the systems development process for users. "This creates a more knowledgeable user community, one who is better able to request and use such technology" (Dodd \& Carr, 1994). User involvement is a mutual benefit process. When users involves in the system development cycle, besides the benefits for developers getting bettering understanding of current system, users also can gain the opportunity to learning and studying the new system, because it is a part of its creation. All of these will contribute to further system implementation and use.

- User involvement provides insights into how individual works impact the departments. Users and data services staff become more attuned to a systems perspective of the whole company that leads to the department integration within the company and work becomes more efficient. Dodd \& Carr (1994) pointed out the close working relationship of data services and users create a consensus of purpose that eliminates the 'we-versus-they' point of view.

With these distinguished advantages, user involvement has been realized as one effective technique for effective methodology implementation. Consequently, the technique is adopted by several system development methodologies.

\section{USER InVOlVement In The Methodology PHASE}

Most of the system development methodologies include initial study phase, analysis phase, design phase, implementation phase and maintenance phase. User involvement always takes in the initial study phase, analysis phase and implementation phase.

\section{A. User involvement in the initial study phase}

Most of the methodologies will request the system developers to invite users to join the initial study phase. The 
users, especially end-users are familiar with the environment where they are working. System developers can get the useful information from users. However, there is a problem that although users have a deep understanding of current environment's problems; they don't know how to represent the problems into systematic language. So, the system developers must make out the essential points from the users opinions.

In this phase, user involvement helps identify the conflict areas of the current environment (Avison \& Fitzgerald, 2003).

\section{B. User involvement in the analysis phase}

In this phase, compared with the initiation phase, user involvement means only the "key" users are involved. The initial study just tries to identify the problems and the conflict areas, developers should to gather and understand information from users in a wider ranger. Thus, the more users involving into the initial study, the more conflict areas can be found in that phase. However analysis phase is quite different. Here system developers need to sum up the problems into system language for the next developing phase. So it isn't necessary to involve all users within the system. Only certain numbers of "Key" users help to give more details of the current system; they also help system developers to make the system documents understandable for users which have not technical background. The definition of "key" users is the people who have the significant effects in their environments (Dodd \& Carr, 1994). For example, the department manager, the team leader, the executive staff are the "key" users.

\section{User involvement in the design phase}

Most of people think that design phase only needs the technical skills of developers instead of e user involvement. However, user involvement still happens in the phase. Users can help system developers build document files as reference for the new system, and they can help the system developers make the balance between the technical aspect and the simplification aspect as well. Moreover, nowadays the companies' situation and the requirements are changeable that lead to the new system drops behind the requirements (Chatzoglou, 1997). Regarding to this problem, system developers must keep contact with users and get latest requirements from them. In this way, system developers can amend their design reasonably for meeting requirements better.

\section{User involvement in the implementation phase and maintenance phase}

Although system developers have tested the system for many times, users have the final right to evaluate whether the new system is satisfactory. So developers have to communicate with users continually and know what they think about the new system. Moreover, developers are responsible for providing education and training for users, which is also a good channel for communication and getting feedback from users.
In the end of the implementation phase, acceptance testing comes to an end when the users feel assured that the new system is running good and it is at this point that the new system becomes fully operational (Isaacs \& Olson 1979).

In the maintenance phase, if users find bugs when they use the system and they should report to the system developers immediately for check. The effective communication can reduce the lost from the bugs. Another aspect is that users may have new requirements about the system; they provide the initial information for the system developers to help them in defining the new problems.

\section{COMPARISON OF USER INVOLVEMENT IN SSM AND SSADM}

SSM (Soft Systems Methodology) is the methodology which focuses on the relationship between the organization and its environment. It uses the soft systems thinking. In soft systems thinking, the objectives of the system are assumed to be more complex than a simple goal that can be achieved or measured. Understanding is achieved in soft systems methods through debate with the users in the system (Avison \& Fitzgerald, 2003).

As for SSADM (Structured Systems Analysis and Design Method), it is a methodology providing developers detailed rules and guidelines for work. It is highly structured, and requires involved users must be very "professional" (Avison \& Fitzgerald, 2003).

\section{A. Comparison of user involvement in the initial study}

In SSM, the initial study phase is to find the unstructured problems. In this phase, system developers will invite the users to join the meeting and find out about the problem situation from as many users in that situation as possible. While talking about with the users, the system developers must act in "user-friendly" in this process (Stivers \& Beard, 1987). Their major object is to help the users to make sure what the conflict areas they are meeting, and who the problem owner is. After that, the second phase for identifying current problem situation in one structured way begins. During the phase, rich pictures are intended to help in problem identification, and the users can understand their situation.

SSADM needs to define the problem in the feasibility study. Users involve in this phase and help the system developers to finish the feasibility report. Feasibility report is based on the users' opinions and the current system investigation (Checkland, 1999).

\section{B. Comparison of user involvement in the analysis phase}

In SSM, after defining problems in the current environment, the next phase is called "Root definition". In this phase, user involvement means users give more details to the system developers. At this stage, debate is most important. The problem solver and the problem owner decide which view to focus on (Avison \& Fitzgerald, 2003).

In SSADM requirements analysis, there are two stages: investigation of current requirements and business system options. Users are the investigation objects in the stages. Users and system developers join together to create the user 
catalogue. Users of the methodology are encouraged to think about the appropriateness of each of the steps.

One aspect in SSADM that is different with the SSM is the users' range. In SSM, since the methodology focuses on the human activities, the user involvement covers all the conflict areas. That means user involvement amount is quite large. It may cause users and system developers feel fuzzy in defining the problems. On the contrary, SSADM requires that users who involve into system development must the deep understanding of the current system, and they have to understand the system thinking language. However, because the user involvement's range is not wide enough, it may lead to the limitation in further new system design and implementation.

\section{CONCLUSION}

"The need to improve systems development quality is increasingly felt by information systems departments in organizations" (Ravichandran \& Rai, 1994). A clear definition of systems development quality is required to focus on quality problems identification in the system. User involvement can support the clear definition, although sometimes the users will give inaccurate information to the system developers. Meaningful user involvement in systems development and an overall user orientation is critical to the success of any development project. User involvement is playing a more and more important role in the system developing cycle.

\section{REFERENCES}

[1] Allen. C.D.et al. User involvement in the design process: why, when \& how? Proceedings of the SIGCHI conference on Human factors in computing systems. ACM press.1993,251-254.
[2] Avison, D. E.,Fitzgerald, G. Information systems development: Methodologies, techniques and tools (3nd edition). Maidenhead, UK: McGraw-Hill,2003.

[3] Barki, H.,Hartwick, J. Rethinking the concept of user involvement. MIS Quarterly, March, 1989, 53-63.

[4] Beyer, H.R.,Holtzblatt, K. Apprenticing with the customer, Communication of the ACM.1995,(6), 45-52.

[5] Carmel, E., Whitaker, R. D.and George, J. F. PD and joint application design: a transatlantic comparison, Communications of the ACM. 1993, (6), 44-48.

[6] Chatzoglou, P. D. Use of methodologies: an empirical analysis of their impact on the economics of the development process, European Journal of Information Systems. 1997, (6), 256-270.

[7] Checkland, P. Soft systems methodology: a 30-year retrospective; Systems thinking, systems practice (New edition). Chichester: John Wiley,1999.

[8] Copenhaver, S. Involving Users in Systems Design. Magazine of Bank Administration. 1986,62(4), 42-48.

[9] Dodd, J. L.,Carr, H. H. Systems development led by end-users. Journal of Systems Management.1994, (45) 8, 34-40.

[10] Fitzgerald, B. Systems Development Methodologies: Time to Advance the Clock, in Systems Development Methods for the Next Century, (Ed W. Wojtkowski), New York:Plenum Press, 1997.

[11] Franz, C. R., Robey,D. Organizational Context, User Involvement, and the Usefulness of Information Systems. Decision Sciences (summer).1986,17(3), 329-356.

[12] Herlea, D. E.User participation in requirements negotiation. [On-line]. Accessed on May 23, 2003 on the World Wide Web: http://igloo.idi.ntnu.no/igroup/proceedings/herlea.pdf.

[13] Isaacs, G. U.,Olson, R. E. Successful Systems are Designed by Users. Infosystems.November,1979, 48.

[14] Ravichandran, T.,Rai, T. The dimensions and correlates of systems development quality. ACM press.1994 (4), 272-282.

[15] Stivers, B. P. Beard, L. H. Information systems: getting back to basics. Journal of Information Management. March,1987, 35-41. 